\title{
Application of the nitrogen laser calibration system in LHAASO-WFCTA
}

\section{Long Chen, ${ }^{a, *}$ Xin Li, ${ }^{a}$ Lisi Ge, ${ }^{b}$ Hu Liu, ${ }^{a}$ Qinning Sun, ${ }^{a}$ Yang Wang, ${ }^{a}$ Junji Xia, ${ }^{a}$ Fengrong Zhu ${ }^{a}$ and Yong Zhang ${ }^{b}$ on behalf of the LHAASO Collaboration}

(a complete list of authors can be found at the end of the proceedings)

${ }^{a}$ School of Physical Science and Technology, Southwest Jiaotong University, No.111, North Section 1, Second Ring Road, Chengdu, China

${ }^{b}$ Key Laboratory of Particle Astrophysics, Institute of High Energy Physics, Chinese Academy of Sciences 19B Yuquan Road,Shijingshan District,Beijing,China

E-mail: longchen@swjtu.edu.cn

The nitrogen laser calibration system for the Wide Field of View Cherenkov Telescope Array (WFCTA) is one of the most important components of the Large High Altitude Air Shower Observatory (LHAASO). This calibration system is composed of three parts, including a highprecision 3D lifting and rotating platform (HiRoP), a nitrogen laser and the wide field of view Cherenkov telescope prototypes. The accuracy of the HiRoP is of great importance for the precise calibration of WFCTA since it controls the laser beam's pointing direction. A home-made HiRoP was introduced and the measurements implies that the angular and lifting accuracies of HiRoP are better than $0.003^{\circ}$ and $0.075 \mathrm{~mm}$, respectively. Furthermore, the pulse energy stability of the nitrogen laser beam is another remarkably important factor. The nitrogen laser system with a wavelength of $337.1 \mathrm{~nm}$ located in a high-precision temperature and humidity's controlling container, resulting in a pulse energy fluctuation less than 3 percent. Besides, the method of a standard zero point verification is employed to improve the long-term accuracy of the beam pointing direction.

$37^{\text {th }}$ International Cosmic Ray Conference (ICRC 2021)

July 12 th - 23rd, 2021

Online - Berlin, Germany

\footnotetext{
*Presenter
} 


\section{Introduction}

As one of the major national science and technology infrastructures, Large High Altitude Air Shower Observatory (LHAASO) is designed to study cosmic rays and gamma rays[1]. The Wide Field-of-view Cherenkov Telescope Array (WFCTA) is one of three detector arrays of LHAASO. WFCTA is an array of Cherenkov fluorescence telescopes, where a movable design idea is adopted. The different array configurations are used for Cherenkov observational mode and fluorescence observational mode to realize the different scientific objects[2]. It aims to measure the spectrum of cosmic rays from $10 \mathrm{TeV}$ to $1 \mathrm{EeV}$ by calculating the number of photons detected. Therefore, the absolute calibration of the number of photons received by WFCTA is the key to obtain the accurate energy of the original cosmic rays.

The nitrogen laser calibration system is widely used in the detectors with Cherenkov/fluorescence observational mode. In the calibration process of LHAASO-WFCTA, the accurate pointing of the laser beam in the all sky is accomplished by the rotating of HiRoP. Therefore, the accuracy of each parameter of HiRoP is critical for that. Pierre Auger Observatory's fluorescence telescope detector array also uses a similar laser calibration system which is capable of providing both a steered beam and a fixed-direction vertical beam. The absolute accuracy of pointing is less than $0.2^{\circ}$, and the relative accuracy is maintained within $1 / 80^{\circ}[3-6,11]$. The absolute direction of the High Resolution Fly's Eyes (HiRes)[7, 10] can point to any azimuth or zenith angle within $0.1^{\circ}$, Relative direction of the mirrors is less than $0.005^{\circ}$ in any azimuth and $0.03^{\circ}$ in any azimuth[8].

In this presentation, the angular and the lifting repeatabilities of HiRoP are better than $0.003^{\circ}$ and $0.075 \mathrm{~mm}$, respectively. And the method of standard zero point is employed to assure the long-time accuracy of the beam pointing direction. The application of thermotank is introduced to improve the energy stability of emitted laser beam to less than $3 \%$.

\section{The components of the nitrogen laser calibration system}

As shown in Figure 1, the nitrogen laser calibration system consists of several components, mainly including HiRoP, the nitrogen laser, the standard zero point, temperature controlling system, timing system, and the programmable logic controller (PLC). The HiRoP, whose precision directly decides the pointing accuracy of the laser,provides the rotation, pitch and elevation of the laser system. The nitrogen laser system, emitting the pulse of $337.1 \mathrm{~nm}$ to calibrate WFCTA, is located on the HiRoP. The standard zero calibration function enables an effective observation of HiRoP's positional accuracy and implements a remote calibration in the case of a misalignment. The temperature controlling system contains several sensors to monitor the temperature and humidity of the environment. And one energy meter is installed to record the laser energy. The timing system, GPS, provides a daily time calibration function for the computer and a pulse trigger signal for the nitrogen laser. PLC, the control system, can not only ensure that HiRoP performance all-day cruise missions stable, but also support more than 20 solutions that can be switched freely.

\section{The standard zero point}

The direction of laser is important because of its relationship with the WFCTA's calibration. After the setup of the nitrogen laser calibration system, we employ the Shadow-Tip Method to find 


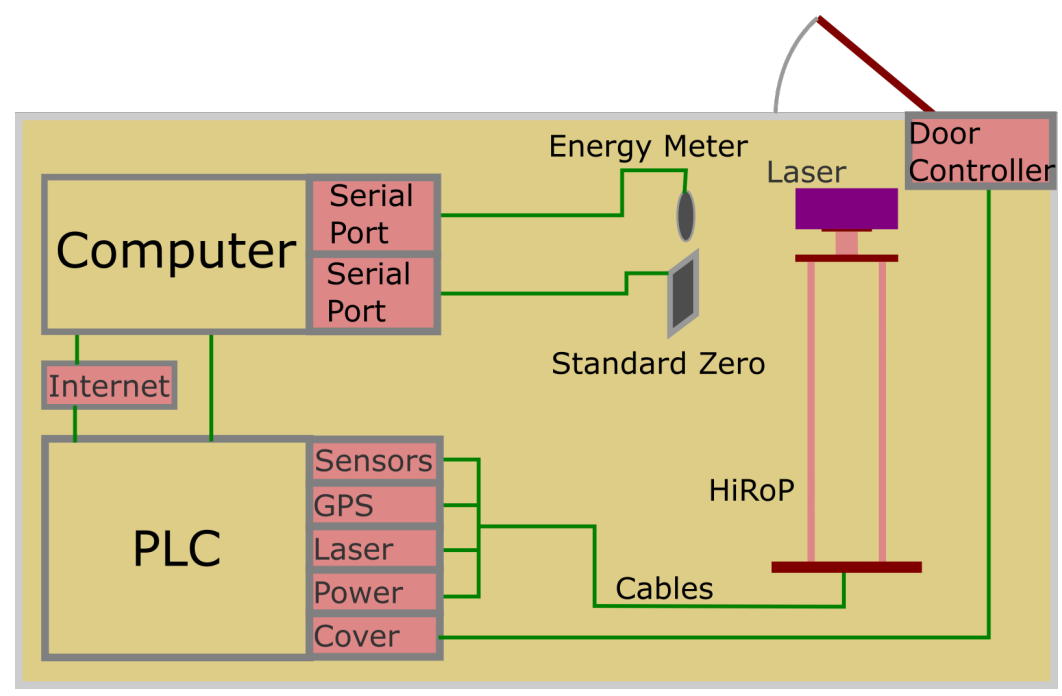

Figure 1: The schematic of the nitrogen laser calibration system

the north direction, and the horizontal direction with gradienter, which are defined as standard zero point. However, long time running may induce some errors of the direction. As shown in Figure 2, special equipment is set up to monitor the standard zero point. The laser is fixed on the top of HiRoP, which produce spots on the coordinate paper fixed on the wall. The distance between the laser and the coordinate paper $L$ is far enough to increase measuring accuracy. The spot is continuously imaged through a CCD camera at a distance of $d$. In order to reduce the influence of background light, dark environment is required when measuring. Meanwhile, to avoid over exposure of the image, a $0.01 \%$ filter is placed in front of the CCD camera. The image of the unexposed light spot is projected horizontally or vertically to obtain the distribution curve of the intensity value in a certain direction. The position coordinates of the spot can be obtained by Mass Center method or the curve fitting with Gaussian function. Each pixel of the CCD camera corresponds to the actual calibration value $\gamma$ by covering the coordinate paper with the field of view of CCD. Therefore, the exact position of the laser spot on the coordinate paper can be obtained. If the spot changes from $\mathrm{O}$ point to A point, the trigonometric function relationship can convert the change of the spot displacement (X pixels) to the angle change of rotation, which can be expressed by $\theta \approx \Delta x / L$. The accuracy can reach $0.001^{\circ}$ by averaged 60 pulse spots when employing nitrogen laser as source light. And if we want to measure the performance of HiRoP, the helium-neon gas laser is more convenient because of its continuous laser. And the accuracy of $0.0009^{\circ}$ can be obtained (Figure $2(b)(c))$.

After 33 days normal running of the laser calibration, the data implies that our system is quite stable, as shown in Figure 3. In the most days, the errors are less than $0.01^{\circ}$, which is the resolution of 0.01 degree monitored by PLC. So Those days can be considered as normal ones. If not, further action is taken to regulate the standard zero point. 


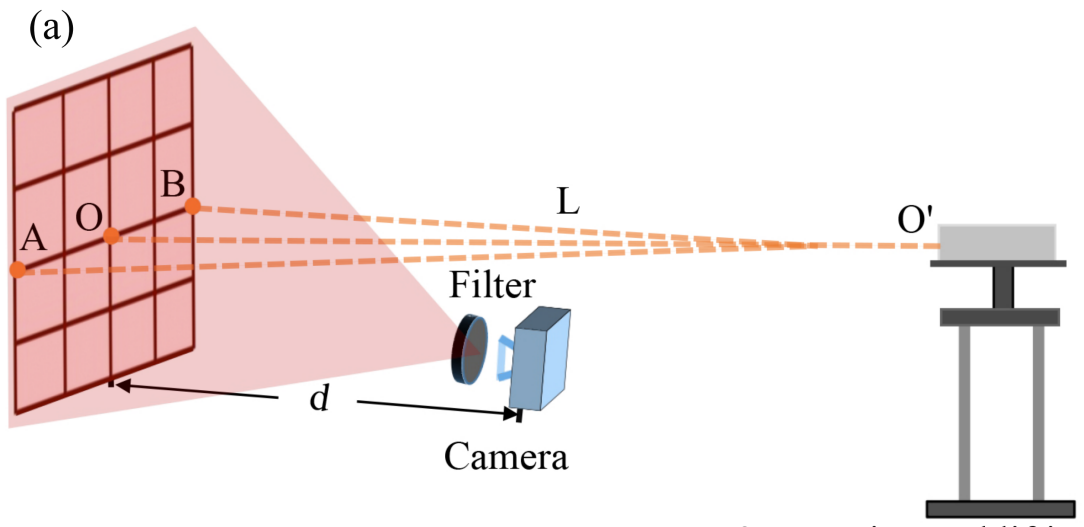

(b)

(c)

$3 \mathrm{D}$ rotating and lifting platform
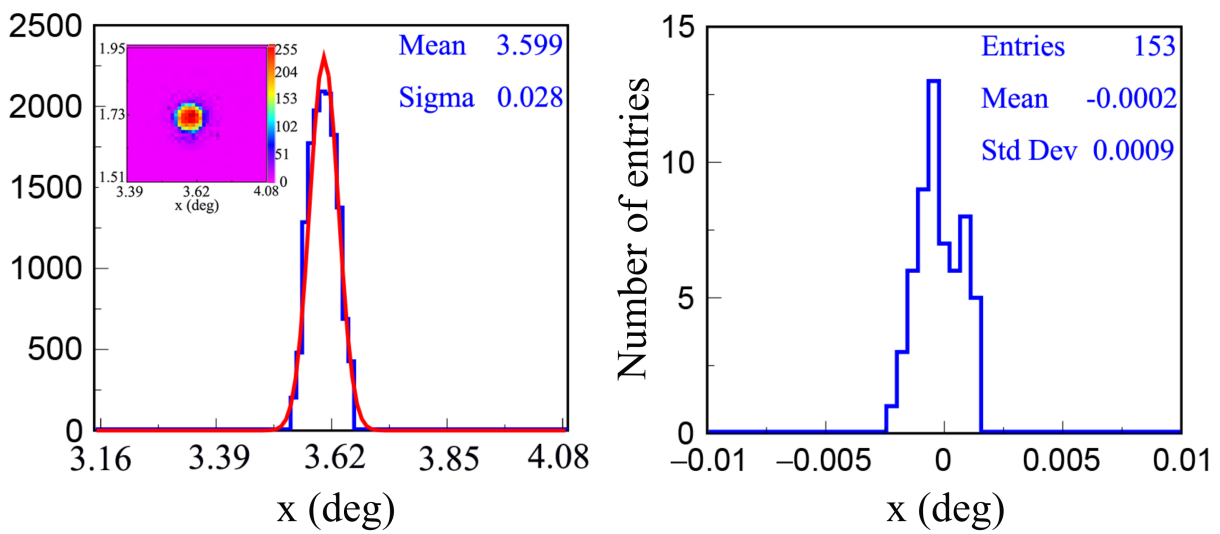

Figure 2: The principle of digital image processing method. (a) Schematic diagram of experimental system construction; (b) The image of the light spot and the projected intensity distribution of the spot on the horizontal axis (blue) and Gaussian fitting curve (red), $x$ axis represents the position of the laser beam projected in the horizontal direction, and $y$ means the intensity of the laser beam corresponding to the $x$; (c) The distribution of laser beam position projected in the horizontal direction.

\section{The performace of HiRoP}

As the source, the helium-neon gas laser can offer continuous and stable gaussian beams, which meets the requirement of measuring the precision of HiRoP. The schematic diagram of measurement principle of HiRoP is the same as the method of standard zero point in Figure 2.The PLC controls the rotation of HiRoP, which is set to a specific angle, named $\Delta \theta$. The laser beam will reach a new position, and the difference between the two positions is the actual rotation angle $\Delta \phi$ of the HiRoP obtained from the method.The HiRoP rotates forward with a certain step, which then stop and run in the opposite direction without doing any operation. As shown in Figure 4, the spot position is linearly fitted. Where $P 0$ is the y-intercept of the fitting straight line, and $P 1$ is the fitted slope value.The closer to 1 the slope value approaches, the better the consistency between the setting value of PLC and the real rotation value measured by the method. The blue dots are the fitting results of the forward data, and the red dots are the fitting results of the reverse data. The difference between the y-intercept values of the two fitting straight lines is rotational error. The back lash of the azimuth direction is $0.004^{\circ}$ degrees in Figure 4 (a). The back lash of pitch direction 


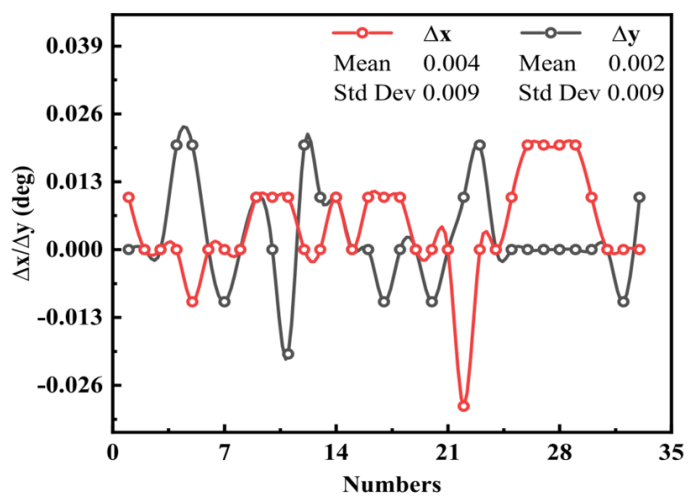

Figure 3: The standard zero position monitoring in horizontal direction and pitch direction with 33 days

is $0.087^{\circ}$ in Figure 4(b). The back lash of lifting direction is better than $0.2 \mathrm{~mm}$ in steps of $2 \mathrm{~mm}$, as shown in Figure 4(c). The slopes of the three fitting straight lines are 0.99, 0.99, 0.98, respectively.
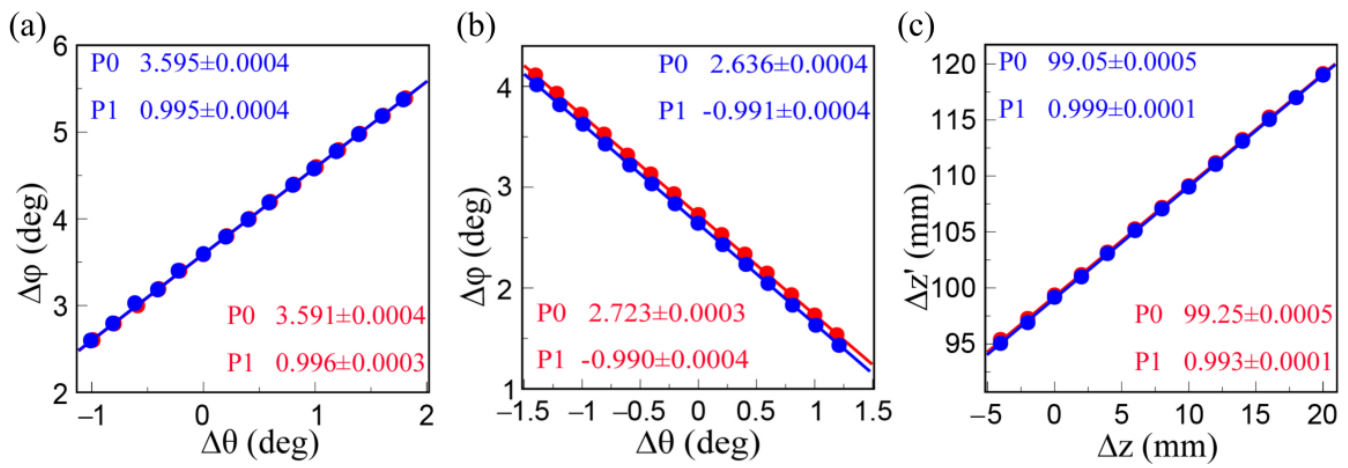

Figure 4: The back lash of the no-load 3D rotating and lifting platform. (a) Horizontal direction; (b) Pitch direction; (c) Lifting direction.

Repeatability refers to the standard deviation value of repeatedly reaching the same position. The 0 position is set to repeat the position multiple times, with the HiRoP rotating $20^{\circ}$ or moving $40 \mathrm{~mm}$ in one dimension, and the statistical distribution of the 0 position is shown in Figure 5. The repeatability of horizontal direction is maintained within $0.003^{\circ}$ in Figure 5(a). The repeatability of pitch direction is less than $0.003^{\circ}$ in Figure 5(b). The repeatability of lifting direction is within $0.058 \mathrm{~mm}$ in Figure 5(c).

\section{The performance of the nitrogen laser}

NL100 nitrogen molecular laser is used in the calibration system as laser source. The wavelength of the laser is $337.1 \mathrm{~nm}$, and the pulse width is $3.5 \mathrm{~ns}$. During the experiment, its operating frequency is $1 \mathrm{~Hz}$, which is triggered by the GPS. The laser provides closed to $170 \mu \mathrm{J}$ each pulse. 

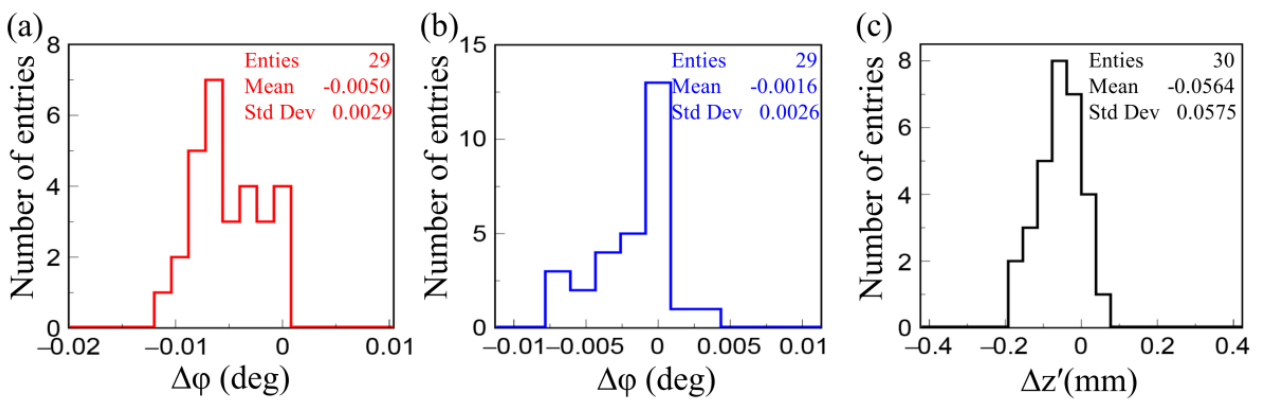

Figure 5: Repeatability and positional accuracy of non-load HiRoP. (a) Horizontal direction; (b) Pitch direction; (c) Lifting direction.

The environment, where the laser works, is harsh that the temperature often changes from 10 degree to -20 degree during one night. That's because the LHAASO site locates at Haizi Mountain, Daocheng County, Sichuan Province, which is the edge of Tibetan Plateau with an altitude up to $4410 \mathrm{~m}$. Although comparing other lasers, the nitrogen laser is sturdy, its energy would decrease as the temperature is too low. Special protections are employed to guarantee its normal working. As shown in Figure 6, thermotank is designed to keep the laser stay in the stable environment with a constant temperature. The thermotank can not only control the temperature, but also prevent the dust or the rain outside.
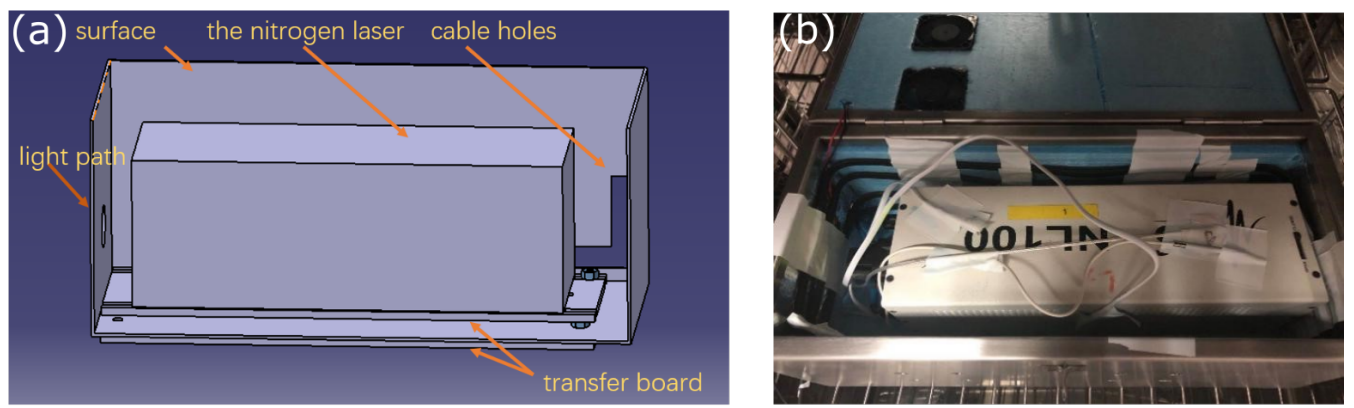

Figure 6: The nitrogen laser and thermotank. (a)the design of thermotank; (b)the diagram of the laser and thermotank.

The energy of the nitrogen laser is directly related to the accuracy of WFCTA calibration. When the laser stores in the thermotank, its energy stays more stable as shown in Figure 7. Removing the first hundreds of second data, the energy stability is about $3 \%$ over 11 hours. During our observation time, the energy of the laser is recorded at the beginning after warming up and at the end of the observation for half an hour. The average energy of the two period predicts the laser energy during the none-measure time.

\section{Discussion}

The calibration system is a complicated system. In this paper, some dominant components of the nitrogen laser calibration system are studied. The high precision of HiRoP is the fundamental 

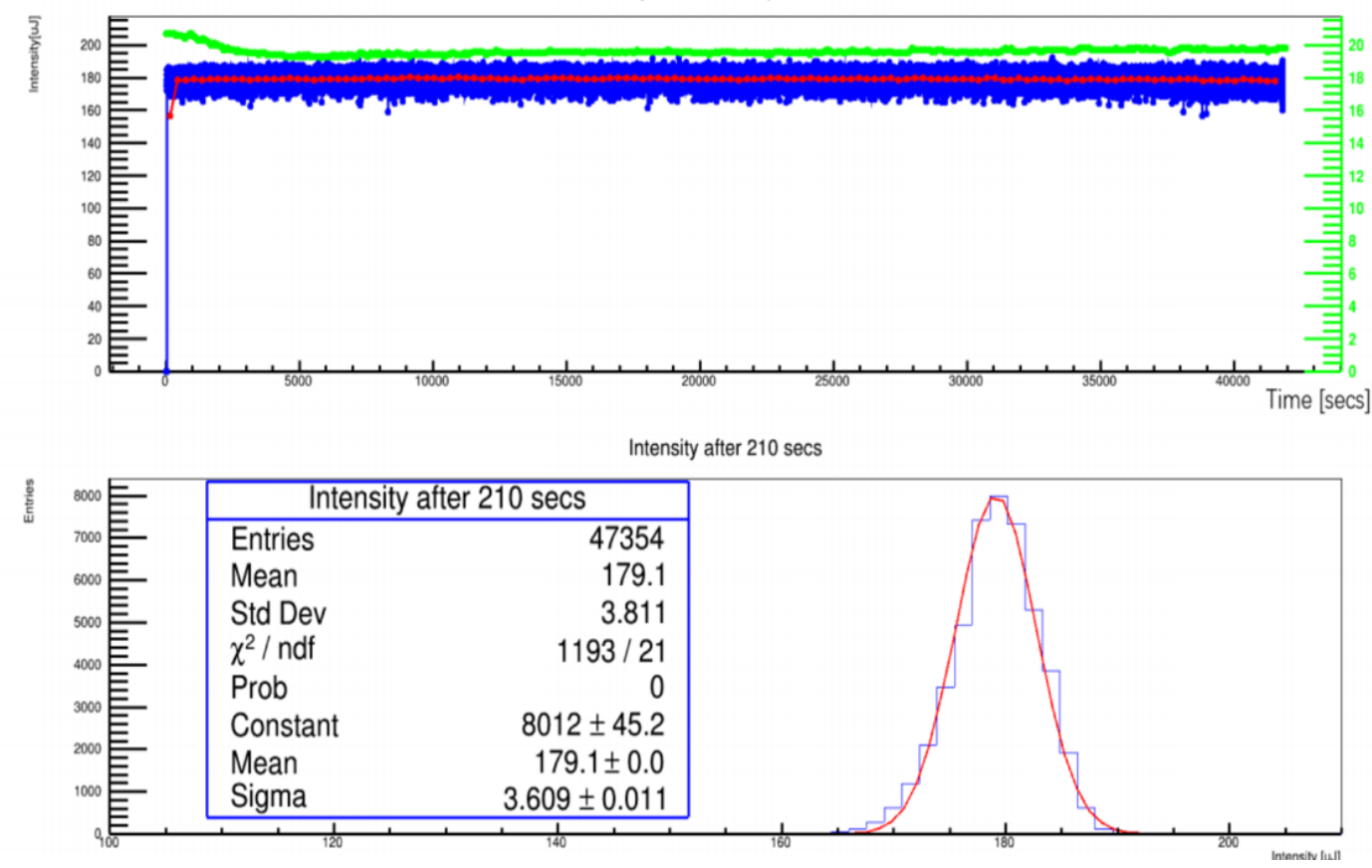

Figure 7: The energy of the nitrogen laser.Top panel: the blue line-dots is the nitrogen energy pulse by pulse, the red line-dots is the average value of one minutes, the green line-dots is the temperature in the thermotank. Bottom panel: the statistical profile of the energy data.

of the pointing accuracy of the laser. The application of the standard zero point assures the long time running. Special protection of the laser provides the energy stability of the laser.

\section{Acknowledgements}

This work is supported by the Science and Technology Department of Sichuan Province (Grant No. 2021YFSY0031, 2020YFSY0016), National Key R\&D program of China under the grants 2018YFA0404201, and also the Fundamental Research Funds for the Central Universities (No. 2682020CX73, 2682020CX74, 2682020CX77).

\section{References}

[1] He, H. (2018). Design of the LHAASO detectors. Radiation Detection Technology and Methods, 2(1), 7.

[2] Zhen, C., et al.. (2019). Introduction to Large High Altitude Air Shower Observatory (LHAASO). Chinese Astronomy and Astrophysics, 43(4), 457-478.

[3] Abraham, J., et al..(2004). Properties and performance of the prototype instrument for the Pierre Auger Observatory. Nucl. Instrum. Methods Phys. Res., Sect. A, 523(1-2), 50-95.

[4] Abraham, J., et al.. (2010). A study of the effect of molecular and aerosol conditions in the atmosphere on air fluorescence measurements at the Pierre Auger Observatory. Astroparticle Physics, 33(2), 108-129. 
[5] Abreu, P., et al.. (2013). Techniques for measuring aerosol attenuation using the Central Laser Facility at the Pierre Auger Observatory. Journal of Instrumentation, 8(4).

[6] Arqueros, F., et al.. (2005). The central laser facility at the Pierre Auger Observatory. 29th International Cosmic Ray Conference, ICRC 2005, 8, 335-338.

[7] Zhang, B., Cao, Z. (2008). The measurement of UHECR spectrum with the HiRes experiment in stereo mode. Nuclear Physics B - Proceedings Supplements, 175-176, 241-244.

[8] Abu-Zayyad, T., et al.. (2000). The prototype high-resolution fly's eye cosmic ray detector. Nucl. Instrum. Methods Phys. Res., Sect. A, 450(2), 253-269.

[9] Liu, J. L., et al. (2018). A calibration of WFCTA prototype telescopes using N2 laser. Nucl. Instrum. Methods Phys. Res., Sect. A, 877, 278-287.

[10] Wiencke, L. R., et al. (1999). Steerable laser system for UV atmospheric monitoring at the High-Resolution Fly's Eye. In G. R. Carruthers K. F. Dymond (Eds.), Ultraviolet Atmospheric and Space Remote Sensing: Methods and Instrumentation II, 3818, 56-63

[11] Mussa, R., et al. (2004). The LIDAR systems for atmospheric monitoring in Auger. Nucl. Instrum. Methods Phys. Res., Sect. A, 518(1-2), 183-185. 


\section{Full Authors List: LHAASO Collaboration}

Note comment afterwards: Collaborations have the possibility to provide an authors list in xml format which will be used while generating the DOI entries making the full authors list searchable in databases like Inspire HEP. For instructions please go to icrc2021.desy.de/proceedings or contact us undericrc2021proc@desy.de.

Zhen $\mathrm{Cao}^{1,2,3}$, F. Aharonian ${ }^{4,5}$, Q. An ${ }^{6,7}$, Axikegu ${ }^{8}$, L.X. Bai ${ }^{9}$, Y.X. Bai ${ }^{1,3}$, L.X. Bai ${ }^{9}$, Y.X. Bai ${ }^{1,3}$, Y.W. Bao ${ }^{10}$, D. Bastieri ${ }^{11}$, X.J. Bi ${ }^{1,2,3}$, Y.J. Bi ${ }^{1,3}$, H. Cai ${ }^{12}$, J.T. Cai ${ }^{11}$, Zhe Cao ${ }^{6,7}$, J. Chang ${ }^{13}$, J.F. Chang ${ }^{1,3,6}$, B.M. Chen ${ }^{14}$, E.S. Chen ${ }^{1,2,3}$, J. Chen ${ }^{9}$, Liang $\mathrm{Chen}^{1,2,3}$, Liang Chen ${ }^{15}$, Long $\mathrm{Chen}^{8}$, M.J. Chen ${ }^{1,3}$, M.L. Chen ${ }^{1,3,6}$, Q.H. Chen ${ }^{8}$, S.H. Chen ${ }^{1,2,3}$, S.Z. Chen ${ }^{1,3}$, T.L. Chen ${ }^{16}$,X.L. Chen $^{1,2,3}$, Y. Chen ${ }^{10}$, N. Cheng ${ }^{1,3}$, Y.D. Cheng ${ }^{1,3}$, S.W. Cui ${ }^{14}$, X.H. Cui ${ }^{17}$, Y.D. Cui ${ }^{18}$, B. D'Ettorre Piazzoli ${ }^{19}$, B.Z. Dai ${ }^{20}$, H.L. Dai $^{1,3,6}$, Z.G. Dai ${ }^{7}$, Danzengluobu ${ }^{16}$, D. della Volpe ${ }^{21}$, X.J. Dong ${ }^{1,3}$, K.K. Duan ${ }^{13}$, J.H. Fan ${ }^{11}$, Y.Z. Fan ${ }^{13}$, Z.X. Fan ${ }^{1,3}$, J. Fang ${ }^{20}$, K. Fang $^{1,3}$, C.F. Feng ${ }^{22}$, L. Feng ${ }^{13}$, S.H. Feng ${ }^{1,3}$, Y.L. Feng ${ }^{13}$, B. Gao ${ }^{1,3}$, C.D. Gao ${ }^{22}$, L.Q. Gao ${ }^{1,2,3}$, Q. Gao ${ }^{16}$, W. Gao ${ }^{22}$, M.M. Ge $^{20}$, L.S. Geng ${ }^{1,3}$, G.H. Gong ${ }^{23}$, Q.B. Gou ${ }^{1,3}$, M.H. Gu${ }^{1,3,6}$, F.L. Guo ${ }^{15}$, J.G. Guo ${ }^{1,2,3}$, X.L. Guo ${ }^{8}$, Y.Q. Guo ${ }^{1,3}$, Y.Y. Guo ${ }^{1,2,3,13}$, Y.A. $\mathrm{Han}^{24}$, H.H. He $\mathrm{H}^{1,2,3}$, H.N. He${ }^{13}$, J.C. He ${ }^{1,2,3}$, S.L. He ${ }^{11}$, X.B. He ${ }^{18}$, Y. He ${ }^{8}$, M. Heller ${ }^{21}$, Y.K. Hor ${ }^{18}$, C. Hou ${ }^{1,3}$, H.B. Hu ${ }^{1,2,3}$, S. $\mathrm{Hu}^{9}$, S.C. $\mathrm{Hu}^{1,2,3}$, X.J. Hu ${ }^{23}$, D.H. Huang ${ }^{8}$, Q.L. Huang ${ }^{1,3}$, W.H. Huang ${ }^{22}$, X.T. Huang ${ }^{22}$, X.Y. Huang ${ }^{13}$, Z.C. Huang ${ }^{8}$, F. Ji ${ }^{1,3}$, X.L. $\mathrm{Ji}^{1,3,6}$, H.Y. Jia ${ }^{8}$, K. Jiang ${ }^{6,7}$, Z.J. Jiang ${ }^{20}$, C. Jin ${ }^{1,2,3}$, T. Ke ${ }^{1,3}$, D. Kuleshov ${ }^{25}$, K. Levochkin ${ }^{25}$, B.B. Li ${ }^{14}$, Cheng Li ${ }^{6,7}$, Cong Li ${ }^{1,3}$,

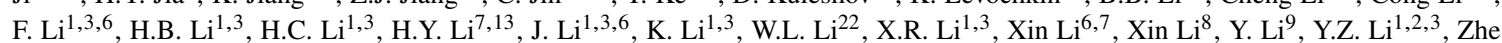

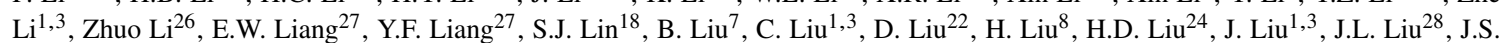

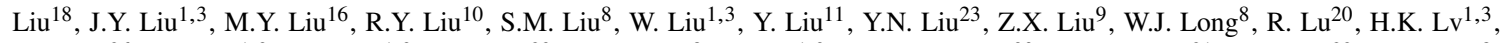
B.Q. $\mathrm{Ma}^{26}$, L.L. $\mathrm{Ma}^{1,3}$, X.H. Ma ${ }^{1,3}$, J.R. $\mathrm{Mao}^{29}$, A. $\operatorname{Masood}^{8}$, Z. $\mathrm{Min}^{1,3}$, W. Mitthumsiri ${ }^{30}$, T. Montaruli ${ }^{21}$, Y.C. Nan ${ }^{22}$, B.Y. Pang ${ }^{8}$, P. Pattarakijwanich ${ }^{30}$, Z.Y. Pei ${ }^{11}$, M.Y. Qi ${ }^{1,3}$, Y.Q. Qi ${ }^{14}$, B.Q. Qiao ${ }^{1,3}$, J.J. Qin ${ }^{7}$, D. Ruffolo ${ }^{30}$, V. Rulev ${ }^{25}$, A. Sáiz ${ }^{30}$, L. Shao ${ }^{14}$, O. Shchegolev $^{25,31}$, X.D. Sheng ${ }^{1,3}$, J.Y. Shi ${ }^{1,3}$, H.C. Song ${ }^{26}$, Yu.V. Stenkin ${ }^{25,31}$, V. Stepanov ${ }^{25}$, Y. Su ${ }^{32}$, Q.N. Sun ${ }^{8}$, X.N. Sun ${ }^{27}$, Z.B. $\mathrm{Sun}^{33}$, P.H.T. Tam ${ }^{18}$, Z.B. Tang ${ }^{6,7}$, W.W. Tian ${ }^{2,17}$, B.D. Wang ${ }^{1,3}$, C. Wang ${ }^{33}$, H. Wang ${ }^{8}$, H.G. Wang ${ }^{11}$, J.C. Wang ${ }^{29}$, J.S. Wang ${ }^{28}$, L.P. Wang $^{22}$, L.Y. Wang ${ }^{1,3}$, R.N. Wang ${ }^{8}$, W. Wang ${ }^{18}$, W. Wang ${ }^{12}$, X.G. Wang ${ }^{27}$, X.J. Wang ${ }^{1,3}$, X.Y. Wang ${ }^{10}$, Y. Wang ${ }^{8}$, Y.D. Wang ${ }^{1,3}$, Y.J. Wang $^{1,3}$, Y.P. Wang ${ }^{1,2,3}$, Z.H. Wang ${ }^{9}$, Z.X. Wang ${ }^{20}$, Zhen Wang ${ }^{28}$, Zheng Wang ${ }^{1,3,6}$, D.M. Wei ${ }^{13}$, J.J. Wei ${ }^{13}$, Y.J. Wei ${ }^{1,2,3}$, T. Wen $^{20}$, C.Y. $\mathrm{Wu}^{1,3}$, H.R. $\mathrm{Wu}^{1,3}$, S. $\mathrm{Wu}^{1,3}$, W.X. Wu ${ }^{8}$, X.F. Wu ${ }^{13}$, S.Q. Xi ${ }^{1,3}$, J. Xia ${ }^{7,13}$, J.J. Xia ${ }^{8}$, G.M. Xiang ${ }^{2,15}$, D.X. Xiao ${ }^{16}$, G. Xiao ${ }^{1,3}$, H.B. Xiao ${ }^{11}$, G.G. Xin ${ }^{12}$, Y.L. Xin ${ }^{8}$, Y. Xing ${ }^{15}$, D.L. Xu ${ }^{28}$, R.X. Xu ${ }^{26}$, L. Xue ${ }^{22}$, D.H. Yan ${ }^{29}$, J.Z. Yan ${ }^{13}$, C.W. Yang ${ }^{9}$, F.F. Yang ${ }^{1,3,6}$, J.Y. Yang ${ }^{18}$, L.L. Yang ${ }^{18}$, M.J. Yang ${ }^{1,3}$, R.Z. Yang ${ }^{7}$, S.B. Yang ${ }^{20}$, Y.H. Yao ${ }^{9}$, Z.G. Yao ${ }^{1,3}$, Y.M. Ye ${ }^{23}$, L.Q. Yin ${ }^{1,3}$, N. Yin ${ }^{22}$, X.H. You $^{1,3}$, Z.Y. You ${ }^{1,2,3}$, Y.H. Yu ${ }^{22}$, Q. Yuan ${ }^{13}$, H.D. Zeng ${ }^{13}$, T.X. Zeng ${ }^{1,3,6}$, W. Zeng ${ }^{20}$, Z.K. Zeng ${ }^{1,2,3}$, M. Zha ${ }^{1,3}$, X.X. Zhai ${ }^{1,3}$, B.B. Zhang $^{10}$, H.M. Zhang ${ }^{10}$, H.Y. Zhang ${ }^{22}$, J.L. Zhang ${ }^{17}$, J.W. Zhang ${ }^{9}$, L.X. Zhang ${ }^{11}$, Li Zhang ${ }^{20}$, Lu Zhang ${ }^{14}$, P.F. Zhang ${ }^{20}$, P.P. Zhang ${ }^{14}$, R. Zhang ${ }^{7,13}$, S.R. Zhang ${ }^{14}$, S.S. Zhang ${ }^{1,3}$, X. Zhang ${ }^{10}$, X.P. Zhang ${ }^{1,3}$, Y.F. Zhang ${ }^{8}$, Y.L. Zhang ${ }^{1,3}$, Yi Zhang ${ }^{1,13}$, Yong Zhang ${ }^{1,3}$, B. Zhao $^{8}$, J. Zhao ${ }^{1,3}$, L. Zhao ${ }^{6,7}$, L.Z. Zhao ${ }^{14}$, S.P. Zhao ${ }^{13,22}$, F. Zheng ${ }^{33}$, Y. Zheng ${ }^{8}$, B. Zhou ${ }^{1,3}$, H. Zhou ${ }^{28}$, J.N. Zhou ${ }^{15}$, P. Zhou ${ }^{10}$, R. Zhou $^{9}$, X.X. Zhou ${ }^{8}$, C.G. Zhu ${ }^{22}$, F.R. Zhu ${ }^{8}$, H. Zhu ${ }^{17}$, K.J. Zhu ${ }^{1,2,3,6}$ and X. Zuo ${ }^{1,3}$

\footnotetext{
${ }^{1}$ Key Laboratory of Particle Astrophyics \& Experimental Physics Division \& Computing Center, Institute of High Energy Physics, Chinese Academy of Sciences, 100049 Beijing, China.

${ }^{2}$ University of Chinese Academy of Sciences, 100049 Beijing, China.

${ }^{3}$ TIANFU Cosmic Ray Research Center, Chengdu, Sichuan, China.

${ }^{4}$ Dublin Institute for Advanced Studies, 31 Fitzwilliam Place, 2 Dublin, Ireland.

${ }^{5}$ Max-Planck-Institut for Nuclear Physics, P.O. Box 103980, 69029 Heidelberg, Germany.

${ }^{6}$ State Key Laboratory of Particle Detection and Electronics, China.

${ }^{7}$ University of Science and Technology of China, 230026 Hefei, Anhui, China.

${ }^{8}$ School of Physical Science and Technology \& School of Information Science and Technology, Southwest Jiaotong University, 610031 Chengdu, Sichuan, China.

${ }^{9}$ College of Physics, Sichuan University, 610065 Chengdu, Sichuan, China.

${ }^{10}$ School of Astronomy and Space Science, Nanjing University, 210023 Nanjing, Jiangsu, China.

${ }^{11}$ Center for Astrophysics, Guangzhou University, 510006 Guangzhou, Guangdong, China.

${ }^{12}$ School of Physics and Technology, Wuhan University, 430072 Wuhan, Hubei, China.

${ }^{13}$ Key Laboratory of Dark Matter and Space Astronomy, Purple Mountain Observatory, Chinese Academy of Sciences, 210023 Nanjing, Jiangsu, China.

${ }^{14}$ Hebei Normal University, 050024 Shijiazhuang, Hebei, China.

${ }^{15}$ Key Laboratory for Research in Galaxies and Cosmology, Shanghai Astronomical Observatory, Chinese Academy of Sciences, 200030 Shanghai, China.

${ }^{16}$ Key Laboratory of Cosmic Rays (Tibet University), Ministry of Education, 850000 Lhasa, Tibet, China.

${ }^{17}$ National Astronomical Observatories, Chinese Academy of Sciences, 100101 Beijing, China.

${ }^{18}$ School of Physics and Astronomy \& School of Physics (Guangzhou), Sun Yat-sen University, 519000 Zhuhai, Guangdong, China.

${ }^{19}$ Dipartimento di Fisica dell'Università di Napoli 'Federico II’, Complesso Universitario di Monte Sant’Angelo, via Cinthia, 80126 Napoli, Italy.
} 
${ }^{20}$ School of Physics and Astronomy, Yunnan University, 650091 Kunming, Yunnan, China.

${ }^{21}$ D'epartement de Physique Nucl'eaire et Corpusculaire, Facult'e de Sciences, Universit'e de Gen 'eve, 24 Quai Ernest Ansermet, 1211 Geneva, Switzerland.

${ }^{22}$ Institute of Frontier and Interdisciplinary Science, Shandong University, 266237 Qingdao, Shandong, China.

${ }^{23}$ Department of Engineering Physics, Tsinghua University, 100084 Beijing, China.

${ }^{24}$ School of Physics and Microelectronics, Zhengzhou University, 450001 Zhengzhou, Henan, China.

${ }^{25}$ Institute for Nuclear Research of Russian Academy of Sciences, 117312 Moscow, Russia.

${ }^{26}$ School of Physics, Peking University, 100871 Beijing, China.

${ }^{27}$ School of Physical Science and Technology, Guangxi University, 530004 Nanning, Guangxi, China.

${ }^{28}$ Tsung-Dao Lee Institute \& School of Physics and Astronomy, Shanghai Jiao Tong University, 200240 Shanghai, China.

${ }^{29}$ Yunnan Observatories, Chinese Academy of Sciences, 650216 Kunming, Yunnan, China.

${ }^{30}$ Department of Physics, Faculty of Science, Mahidol University, 10400 Bangkok, Thailand.

${ }^{31}$ Moscow Institute of Physics and Technology, 141700 Moscow, Russia.

${ }^{32}$ Key Laboratory of Radio Astronomy, Purple Mountain Observatory, Chinese Academy of Sciences, 210023 Nanjing, Jiangsu, China.

${ }^{33}$ National Space Science Center, Chinese Academy of Sciences, 100190 Beijing, China. 